\title{
Malnutrition in patients admitted to in-hospital cardiac rehabilitation: Clinical correlates and association with mortality
}

\author{
Andrea Passantino, Pietro Guida, Caterina Rizzo, Rosa Carbonara, Roberta Ruggieri, Domenico Scrutinio \\ Istituti Clinici Scientifici Mangeri IRCCS, Institute of Bari, Italy
}

\section{Abstract}

Malnutrition is highly prevalent among hospitalized patients; thus, an accurate identification of malnutrition could improve the outcome of these patients. The aim of the present paper was to apply multiple methods to evaluate the prevalence of malnutrition and clinical correlates in patients admitted to in-hospital cardiac rehabilitation. We performed a prospective study of 426 patients admitted to in-hospital cardiac rehabilitation: $282(66.2 \%)$ had undergone a major cardiac surgery and 144 (34.8\%) had experienced heart failure. The albumin level and mini nutritional assessment (MNA) scores were applied to evaluate the nutritional status

Correspondence: Andrea Passantino MD, Cardiology Department, ICS Maugeri IRCCS, Institute of Bari, Via Generale Bellomo 73/75, Bari, Italy.

Tel. +39.080 .7814293 - Fax: +39.080 .7814280$

E-mail: andrea.passantino@icsmaugeri.it

Key words: malnutrition; cardiac rehabilitation; heart failure; cardiac surgery; Mini Nutritional Assessment.

Contributions: AP, PG and DS contributed to the conception or design of the work. PG, RC, RR and CR contributed to the acquisition, analysis, or interpretation of data for the work. AP and PG drafted the manuscript. DS, RC, CS and RR critically revised the manuscript. All gave final approval and agree to be accountable for all aspects of work ensuring integrity and accuracy.

Conflict of interest: The authors have no competing interests to declare.

Funding: This research did not receive any specific grant from funding agencies in the public, commercial, or not-for-profit sectors.

Ethical approval and consent to participate: The study was approved by the local Ethics Committee. All patients gave informed consent to participate in the study. This study was conducted in accordance with the Declaration of Helsinki.

Received for publication: 1 June 2021.

Accepted for publication: 5 October 2021.

${ }^{\circ}$ Copyright: the Author(s), 2021

Licensee PAGEPress, Italy

Monaldi Archives for Chest Disease 2022; 92:1960

doi: 10.4081/monaldi.2021.1960

This article is distributed under the terms of the Creative Commons Attribution Noncommercial License (by-nc 4.0) which permits any noncommercial use, distribution, and reproduction in any medium, provided the original author(s) and source are credited. of these patients. Serum albumin levels were $<3.5 \mathrm{~g} / \mathrm{dl}$ in 147 (34.5\%) patients, and MNA scores were < 24 in $179(42.0 \%)$ patients. Patients with malnutrition or a risk of malnutrition had lower haemoglobin values, lower EuroQol scores and poorer functional status. Female gender, age, functional status and cumulative illness rating scale severity were predictors of malnutrition. Over a median follow-up of 47 months, MNA scores $<24$ were associated with higher mortality, even after correction for confounding variables. In conclusion, in patients admitted to in-hospital cardiac rehabilitation, malnutrition and risk of malnutrition frequently occur and are associated with poor functional status, higher clinical complication rates and long-term mortality.

\section{Introduction}

Cardiac rehabilitation is a comprehensive multifactorial intervention for patients who have experienced a recent myocardial infarction, heart failure or cardiac surgery. Rehabilitative programmes involve exercise training, advice on lifestyle modifications, risk factor management and psychosocial support.

In-hospital rehabilitation is dedicated to patients with a more severely impaired functional status or high-risk clinical profile; in this subgroup of patients, which often includes frail and elderly subjects, compromised nutritional status may affect functional and clinical outcomes.

The estimated prevalence of malnutrition in hospitalized patients has been reported at 20-50\%; however, in long-term facilities, the percentage of malnutrition may be as high as $85 \%$ [1-4]. Malnutrition is associated with poor in-hospital outcomes and increased risks of major and minor complications [5-7]; moreover, malnutrition is correlated with increases in direct and indirect costs $[8,9]$.

Notwithstanding its clinical relevance, malnutrition lacks a common, unified definition, and there is uncertainty regarding the optimal method for screening and diagnosing malnourished patients. A variety of methods is available for evaluating malnutrition, but a gold standard has not yet been determined. Tools used to evaluate nutritional status include anthropometric measures, questionnaires and laboratory variables. Thus, assessments of malnutrition prevalence, clinical correlates and prognostic implications may differ depending on the measurement technique.

The aim of the present paper was to apply the albumin dosage and mini nutritional assessment (MNA) to evaluate the prevalence of malnutrition (or risk of malnutrition) and its clinical correlates in patients admitted to in-hospital cardiac rehabilitation for heart failure or recent cardiac surgery. We calculated the agreement between the albumin and MNA results in identifying malnutrition and evaluated the prognostic value of malnutrition in both heart failure and cardiac surgery patients. 


\section{Materials and Methods}

\section{Study population}

We enrolled patients consecutively admitted to the Cardiology Unit of Fondazione S. Maugeri, Cassano delle Murge, Bari, Italy, for in-hospital cardiac rehabilitation. We recruited patients who were $\geq 18$ years old, with the following inclusion criteria: i) cardiac surgery within two months prior to admission; or ii) chronic heart failure with a reduced ejection fraction (evidence of left ventricular ejection fraction $<45 \%$ ) in NYHA class III-IV. The following exclusion criteria were applied: active neoplastic disease and inability to provide informed consent due to cognitive impairment.

At admission, clinical evaluation, echocardiographic examination, nutritional status evaluation, functional assessment, comorbidity evaluation and laboratory measurements were performed for each patient. Nutritional status was evaluated by anthropometric measures, MNA and laboratory measurements.

\section{Nutritional status}

The MNA is a screening tool aimed at assessing nutritional status in elderly patients through 18 questions in 4 areas (basic anthropometrics, dietary intake, global indicators and self-assessed health status) [10]. Individuals with a score of 24-30 are considered to have a normal nutritional status, a score of 17-23.5 suggests a risk of malnutrition, and a score of $<17$ identifies malnutrition [11,12].

Albumin levels were determined using a Bromocresol Green assay kit (Cobas c 311 Clinical Chemistry Analyser, Roche Diagnostics). According to previous reports, a cut-off level of 3.5 $\mathrm{g} / \mathrm{dl}$ albumin was used to identify malnutrition [13].

\section{Anthropometric measures}

The patients were weighed before breakfast without their shoes, and the body mass index (BMI) was calculated as [weight $(\mathrm{kg})] /[\text { height }(\mathrm{m})]^{2}$.

\section{Laboratory}

Complete blood counts and creatinine, haemoglobin, albumin, lymphocyte and serum iron levels were measured. The glomerular filtration rate (eGFR) was estimated by the Cockcroft-Gault formula. Anaemia was classified according to World Health Organization criterion of a haemoglobin level lower than $13.0 \mathrm{~g} / \mathrm{dl}$ in men and $12.0 \mathrm{~g} / \mathrm{dl}$ in women, with values of less than $11.0 \mathrm{~g} / \mathrm{dl}$ corresponding to moderate-to-severe cases in both sexes [14].

\section{Functional assessment}

The functional status at admission was evaluated based on the 6-min walking test (6MWT), which was performed in an indoor 60 -m-long corridor, according to the recommendations of the American Thoracic Society [15]. All patients were instructed to walk along the corridor from one end to the other at their own pace, as many times as possible, in the permitted time. After $6 \mathrm{~min}$ had elapsed, the patients were instructed to stop walking, and the total distance walked was determined. This test was supervised by a physical therapist who encouraged the patients in a standardized fashion at regular intervals.

For patients unable to walk or participate in the 6MWT, the functional status was evaluated by means of the Barthel index [16]. The Barthel index is based on an ordinal scale for ten activities of daily living. The score ranges from 0 , representing a totally dependent bedridden state, to 100 , representing complete independence.

\section{EuroQol}

The EuroQol is a measure of self-reported health outcomes, based on a visual analogue graduated scale (0 to 100) for assessing health status, ranging from the worst imaginable health state $(0)$ to the best imaginable health state $(100)[17,18]$.

\section{Cumulative illness rating scale}

Pre-existing comorbidities were assessed using the cumulative illness rating scale (CIRS) [19], which gives a severity score and comorbidity score. This index is based on the scoring (from 0 to 4 ) of disease severity for 14 items corresponding to organs that may be affected by chronic disease. The CIRS severity score can be calculated as the average of each CIRS item score. The CIRS comorbidity score is based on the count of organ systems with moderateto-severe impairment.

\section{Follow-up}

For patients residing in the Apulia region, the vital status was ascertained through the regional Health Information System, and live patients were determined at the end of the follow-up period (July $31^{\text {st }}, 2019$ ). Patients from regions other than Apulia were excluded from survival analyses.

\section{Statistical analysis}

The data are shown as the mean value \pm standard deviation, and categorical variables are given as frequencies or percentages. Continuous variables were compared by using Student's $t$-test or the Mann-Whitney U-test for variables with a non-normal distribution. Associations between categorical data were evaluated by $\chi^{2}$ or Fisher's exact test, as appropriate. The percentages of patients with albumin levels $<3.5 \mathrm{~g} / \mathrm{dl}$ and MNA scores $<24$ points were calculated to determine the agreement. The Pearson correlation coefficient was used to measure the strength of linear associations among variables. Multivariate logistic regression analyses with a backward stepwise elimination approach were implemented ( $p>0.05$ for removal) to assess independent determinants of malnutrition. The stepwise procedures evaluated the following predictors: age, gender, BMI, heart rate, systolic blood pressure, diabetes, previous stroke, history of myocardial infarction, oxygen saturation, eGFR, ejection fraction, CIRS severity and comorbidity and 
poor mobility (inability to participate in the 6MWT). For cardiac surgery patients, we also evaluated the time from cardiac surgery to admission for rehabilitation, the occurrence of a combined cardiac surgery and the incidence of early complications during the hospital stay in which the operation was performed. The odds ratios (ORs) for malnutrition and corresponding 95\% confidence intervals $(95 \% \mathrm{CIs})$ are reported. The model discrimination was evaluated via the area under the receiver operating characteristic curve (AUROC). Mortality curves were generated with the Kaplan-Meier method and were compared based on malnutrition status using the log-rank test. A Cox proportional hazards model was evaluated to derive hazard ratios (HRs) with 95\% CIs for allcause mortality, and p-values $<0.05$ were considered statistically significant. The analyses were performed using STATA software, version 14 (StataCorp, College Station, TX, USAas).

\section{Results}

\section{Study population}

From September 2014 to April 2016, 426 patients were enrolled in the study; 144 (34.8\%) had experienced heart failure, and $282(66.2 \%)$ were admitted after cardiac surgery. No patients died during the hospital stay (20 \pm 7 days). For surgical patients, the duration between admission and rehabilitation was $13 \pm 9$ days. Combined cardiac procedures were reported for 43 patients
$(15.3 \%)$, and early complications after surgery (before referral to cardiac rehabilitation) were experienced in 38 patients (13.5\%).

Table 1 shows the patient characteristics for the overall population and by group. Serum albumin levels were lower than $3.5 \mathrm{~g} / \mathrm{dl}$ in $147(34.5 \%)$ patients: $138(48.9 \%)$ in the cardiac surgery group and $9(6.3 \%)$ in the heart failure group. Among the overall study population, MNA scores were $<24$ in 179 patients (42.0\%), while only 8 patients $(1.9 \%)$ had an MNA score $<17$. The MNA score was significantly lower in heart failure patients compared with cardiac surgery patients.

Table 2 shows the patient clinical characteristics by malnutrition parameters: albumin $<3.5 \mathrm{~g} / \mathrm{dl}$ or MNA score $<24$. In heart failure patients, low albumin levels were correlated with moderateto-severe anaemia, and low MNA scores were associated with lower EuroQol values. In cardiac surgery patients, albumin levels $<3.5 \mathrm{~g} / \mathrm{dl}$ were associated with peripheral oedema, pleural effusion and lower haemoglobin, serum iron, total cholesterol and EuroQol values. Patients with MNA scores $<24$ had lower EuroQol values. In both groups, a smaller percentage of patients with albumin levels $<3.5 \mathrm{mg} / \mathrm{dl}$ and MNA scores $<24$ were able to perform the $6 \mathrm{MWT}$. EuroQol values were significantly lower in patients with MNA scores $<24$, in both the heart failure and cardiac surgery groups. The proportion of patients with both an albumin level $<3.5$ $\mathrm{mg} / \mathrm{dl}$ and MNA score $<24$ was $50 \%$ in the overall population and in each subgroup.

In backward stepwise elimination multivariate logistic regression analyses (Table 3 ), no predictors were identified in heart fail-

Table 1. Patients' characteristics by groups.

\begin{tabular}{|c|c|c|c|c|}
\hline & $\begin{array}{c}\text { All } \\
\mathrm{n}=426\end{array}$ & $\begin{array}{c}\text { Heart failure } \\
n=144\end{array}$ & $\begin{array}{c}\text { Cardiac surgery } \\
n=282\end{array}$ & p \\
\hline Age (years) & $67 \pm 11$ & $67 \pm 12$ & $67 \pm 10$ & 0.501 \\
\hline Male gender & $296(69.5 \%)$ & $110(76.4 \%)$ & $186(66.0 \%)$ & 0.027 \\
\hline BMI $\left(\mathrm{Kg} / \mathrm{m}^{2}\right)$ & $29 \pm 5$ & $29 \pm 6$ & $28 \pm 4$ & 0.105 \\
\hline Diabetes & $139(32.6 \%)$ & $51(35.4 \%)$ & $88(31.2 \%)$ & 0.381 \\
\hline Peripheral edema & $55(12.9 \%)$ & $23(16.0 \%)$ & $32(11.3 \%)$ & 0.178 \\
\hline Pleural effusion & $182(42.7 \%)$ & $21(14.6 \%)$ & $161(57.1 \%)$ & $<0.001$ \\
\hline Hemoglobin ( $\mathrm{g} / \mathrm{dl})$ & $11.1 \pm 1.8$ & $12.6 \pm 2.0$ & $10.4 \pm 1.2$ & $<0.001$ \\
\hline Anemia & $343(80.5 \%)$ & $72(50.0 \%)$ & $271(96.1 \%)$ & $<0.001$ \\
\hline Serum iron (mcg/dl) & $48 \pm 31$ & $69 \pm 40$ & $38 \pm 18$ & $<0.001$ \\
\hline eGFR (ml/min/1.73 m²) & $70 \pm 23$ & $62 \pm 24$ & $73 \pm 22$ & $<0.001$ \\
\hline $\mathrm{eGFR}<60 \mathrm{ml} / \mathrm{min} / 1.73 \mathrm{~m}^{2}$ & $158(37.1 \%)$ & $70(48.6 \%)$ & $88(31.2 \%)$ & $<0.001$ \\
\hline Total cholesterol (mg/dl) & $145 \pm 39$ & $145 \pm 42$ & $145 \pm 37$ & 0.915 \\
\hline Ejection fraction (\%) & $47 \pm 13$ & $35 \pm 12$ & $53 \pm 8$ & $<0.001$ \\
\hline Ejection fraction $\leq 35 \%$ & $100(23.5 \%)$ & $88(61.1 \%)$ & $12(4.3 \%)$ & $<0.001$ \\
\hline CIRS severity & $1.47 \pm 0.23$ & $1.54 \pm 0.27$ & $1.43 \pm 0.20$ & $<0.001$ \\
\hline CIRS comorbidity & $2.4 \pm 1.2$ & $2.7 \pm 1.5$ & $2.2 \pm 1.1$ & $<0.001$ \\
\hline EuroQol & $48 \pm 15$ & $48 \pm 16$ & $48 \pm 15$ & 0.920 \\
\hline 6MWT not performed & $175(41.1 \%)$ & $53(36.8 \%)$ & $122(43.3 \%)$ & 0.200 \\
\hline 6MWT (m) & $315 \pm 84$ & $331 \pm 92$ & $305 \pm 78$ & 0.016 \\
\hline Barthel index & $67 \pm 16$ & $68 \pm 19$ & $67 \pm 14$ & 0.663 \\
\hline Albumin (g/dl) & $3.71 \pm 0.47$ & $4.07 \pm 0.44$ & $3.53 \pm 0.37$ & $<0.001$ \\
\hline Albumin $<3.5 \mathrm{~g} / \mathrm{dl}$ & $147(34.5 \%)$ & $9(6.3 \%)$ & $138(48.9 \%)$ & $<0.001$ \\
\hline MNA & $23.9 \pm 3.3$ & $23.1 \pm 3.6$ & $24.4 \pm 3.1$ & $<0.001$ \\
\hline MNA $<24$ & $179(42.0 \%)$ & $74(51.4 \%)$ & $105(37.2 \%)$ & 0.005 \\
\hline
\end{tabular}

Mean \pm SD and frequency (percentage). 
ure patients for albumin levels $<3.5 \mathrm{~g} / \mathrm{dl}$, while MNA scores $<24$ were associated with female gender and CIRS severity (AUROC: $0.65)$.

In cardiac surgery patients, albumin levels $<3.5 \mathrm{~g} / \mathrm{dl}$ were predicted by age, time from cardiac surgery to rehabilitation and inability to perform the 6MWT (AUROC: 0.69). The likelihood of an MNA score $<24$ was associated with the occurrence of early complications after surgery, lower BMI and inability to perform the 6MWT (AUROC: 0.68). Figure 1 shows scatter plots of MNA scores and albumin levels for both groups. Albumin levels were correlated with MNA scores in heart failure patients (Figure 1A) but not in cardiac surgery patients (Figure 1B).

Follow-up was performed for 406 patients residing in the Apulia region (140 heart failure and 266 cardiac surgery patients). After a median follow-up of 47 months (interquartile range: 41-54 months), 77 patients died (overall cumulative mortality at 48 months: $19.3 \%$ ): 51 in the heart failure group (cumulative mortality at 48 months: $37.9 \%$ ) and 26 in the cardiac surgery group (cumulative mortality at 48 months: 9.6\%). Patients with an MNA score $<24$ exhibited significantly higher mortality in comparison to patients with MNA scores $\geq 24$ (log-rank test $\mathrm{p}=0.027$, cumulative mortality at 48 months: $24.7 \%$ vs $15.2 \%$ ) (Figure 2). Each MNA point below 24 significantly increased the mortality, with $\mathrm{HR}=$
1.15 (95\% CI: 1.07-1.23; $\mathrm{p}<0.001)$ for Cox univariate analysis and $\mathrm{HR}=1.12(95 \% \mathrm{CI}: 1.03-1.22 ; \mathrm{p}=0.008)$ adjusting for age, sex and study group. In the cardiac surgery group, albumin levels $<3.5$ $\mathrm{g} / \mathrm{dl}$ were not associated with mortality (log-rank test $\mathrm{p}=0.972$, cumulative mortality at 48 months: $9.2 \%$ vs $10.0 \%$, respectively, for patients with levels $<3.5 \mathrm{~g} / \mathrm{dl}$ and $\geq 3.5 \mathrm{~g} / \mathrm{dl}$ ). For the heart failure patients, the association of low albumin with mortality was not evaluated.

\section{Discussion}

In the present study, we evaluated the prevalence of malnutrition and risk of malnutrition in a cohort of 426 patients admitted to in-hospital rehabilitation after heart surgery or heart failure. In the overall study population, we found MNA scores $<24$ in $42 \%$ of patients. This cut-off identifies patients with both malnutrition and malnutrition risk; however, only eight of these patients were confirmed as malnourished (MNA <17). This low frequency may be due to the low sensitivity of the MNA in this care setting. Female gender, CIRS severity and inability to perform the 6MWT were identified as predictors for MNA scores $<24$.

Table 2. Patients' clinical characteristics by malnutrition parameters.

\begin{tabular}{|c|c|c|c|c|c|c|}
\hline Heart failure & $\begin{array}{c}\text { No } \\
\mathrm{n}=135\end{array}$ & $\begin{array}{c}\text { umin }<3.5 \\
\text { Yes } \\
n=9\end{array}$ & p & $\begin{array}{c}\text { No } \\
n=70\end{array}$ & $\begin{array}{c}\text { NA }<24 \text { poi } \\
\text { Yes } \\
n=74\end{array}$ & p \\
\hline Peripheral edema & $20(14.8 \%)$ & $3(33.3 \%)$ & 0.156 & $13(18.6 \%)$ & $10(13.5 \%)$ & 0.408 \\
\hline Pleural effusion & $18(13.3 \%)$ & $3(33.3 \%)$ & 0.126 & $9(12.9 \%)$ & $12(16.2 \%)$ & 0.568 \\
\hline Hemoglobin ( $\mathrm{g} / \mathrm{dl})$ & $12.7 \pm 2.0$ & $11.7 \pm 2.3$ & 0.138 & $12.8 \pm 2.1$ & $12.5 \pm 1.9$ & 0.372 \\
\hline Anemia & $65(48.1 \%)$ & $7(77.8 \%)$ & 0.166 & $34(48.6 \%)$ & $38(51.4 \%)$ & 0.739 \\
\hline Moderate-to severe anemia & $30(22.2 \%)$ & $6(66.7 \%)$ & 0.008 & $16(22.9 \%)$ & $20(27.0 \%)$ & 0.564 \\
\hline Lymphocytes (10E9/1) & $1.88 \pm 0.85$ & $1.33 \pm 0.71$ & 0.082 & $1.86 \pm 0.79$ & $1.83 \pm 0.90$ & 0.808 \\
\hline Serumiron (mcg/dl) & $70 \pm 39$ & $60 \pm 46$ & 0.164 & $73 \pm 35$ & $66 \pm 44$ & 0.339 \\
\hline Total cholesterol (mg/dl) & $146 \pm 43$ & $132 \pm 24$ & 0.338 & $148 \pm 45$ & $143 \pm 39$ & 0.489 \\
\hline EuroQol & $49 \pm 16$ & $42 \pm 15$ & 0.213 & $55 \pm 16$ & $41 \pm 13$ & $<0.001$ \\
\hline 6MWT not perfomed & $47(34.8 \%)$ & $6(66.7 \%)$ & 0.075 & $19(27.1 \%)$ & $34(45.9 \%)$ & 0.019 \\
\hline 6MWT (m) & $331 \pm 93$ & $340 \pm 35$ & 0.869 & $351 \pm 95$ & $306 \pm 81$ & 0.018 \\
\hline Barthel index & $68 \pm 20$ & $65 \pm 14$ & 0.490 & $78 \pm 12$ & $62 \pm 21$ & 0.002 \\
\hline Cardiacsurgery & $\begin{array}{c}\text { No } \\
\mathrm{n}=144\end{array}$ & $\begin{array}{c}\text { umin }<3.5 \\
\text { Yes } \\
n=138\end{array}$ & p & $\begin{array}{c}\text { No } \\
\mathrm{n}=177\end{array}$ & $\begin{array}{c}\text { NA }<24 \text { poi } \\
\text { Yes } \\
n=105\end{array}$ & p \\
\hline Peripheral edema & $6(4.2 \%)$ & $26(18.8 \%)$ & $<0.001$ & $20(11.3 \%)$ & $12(11.4 \%)$ & 0.974 \\
\hline Pleural effusion & $70(48.6 \%)$ & $91(65.9 \%)$ & 0.003 & $111(62.7 \%)$ & $50(47.6 \%)$ & 0.013 \\
\hline Hemoglobin ( $\mathrm{g} / \mathrm{dl})$ & $10.9 \pm 1.1$ & $9.8 \pm 1.0$ & $<0.001$ & $10.3 \pm 1.1$ & $10.5 \pm 1.3$ & 0.093 \\
\hline Anemia & $134(93.1 \%)$ & $137(99.3 \%)$ & 0.007 & $171(96.6 \%)$ & $100(95.2 \%)$ & 0.545 \\
\hline Moderate-to severe anemia & $75(52.1 \%)$ & $123(89.1 \%)$ & $<0.001$ & $132(74.6 \%)$ & $66(62.9 \%)$ & 0.037 \\
\hline Lymphocytes (10E9/1) & $1.98 \pm 0.79$ & $1.85 \pm 0.90$ & 0.199 & $1.95 \pm 0.91$ & $1.86 \pm 0.74$ & 0.385 \\
\hline Serumiron (mcg/dl) & $43 \pm 20$ & $32 \pm 12$ & $<0.001$ & $36 \pm 16$ & $40 \pm 20$ & 0.055 \\
\hline Total cholesterol (mg/dl) & $158 \pm 40$ & $132 \pm 29$ & $<0.001$ & $142 \pm 34$ & $151 \pm 42$ & 0.058 \\
\hline EuroQol & $51 \pm 16$ & $45 \pm 13$ & 0.001 & $50 \pm 15$ & $46 \pm 15$ & 0.031 \\
\hline 6MWT not perfomed & $48(33.3 \%)$ & $74(53.6 \%)$ & 0.001 & $65(36.7 \%)$ & $57(54.3 \%)$ & 0.004 \\
\hline 6MWT (m) & $310 \pm 79$ & $297 \pm 75$ & 0.278 & $302 \pm 76$ & $312 \pm 82$ & 0.436 \\
\hline Barthel index & $69 \pm 12$ & $65 \pm 15$ & 0.218 & $68 \pm 14$ & $65 \pm 15$ & 0.252 \\
\hline
\end{tabular}

Mean \pm SD and frequency (percentage). 
We found that malnutrition and risk of malnutrition are associated with poor functional status at admission and with higher rates of clinical complications such as anaemia, peripheral oedema and iron deficiency. Furthermore, malnutrition is associated with poor long-term prognoses.

We identified clinical predictors of malnutrition and the differences in prevalence between two groups: heart failure and cardiac surgery patients. The poor agreement among different screening tools suggests the need for an integrated approach for comprehensively classifying malnutrition. The reduced food intake and catabolic state, with a corresponding loss of lean mass, induced by inflammation are possible physio-pathological mechanisms involved in malnutrition in patients who have experienced cardiac surgery or heart failure.

An inadequate caloric supply due to hospital diet, iatrogenic lack of appetite, visceral congestion, eating-induced dyspnoea and difficulty swallowing due to orotracheal intubation in the postsurgical status are other possible causes of malnutrition.

Proinflammatory factors, with increased levels of cytokines or necrohormones, may increase protein catabolism, inducing the downregulation of protein production.

After cardiac surgery, inflammation is accompanied by free radical production, loss of antioxidant substances and transitory reductions in the plasmatic concentration of leucites and vitamins A, B and E [20].

\section{Heart failure patients}

The prevalence of malnutrition in hospitalized patients with heart failure ranges from $34 \%$ to $70 \%$ [21]. Differences in the criteria used to define malnutrition and in the characteristics of study populations may explain this large variance. In our study, the prevalence of MNA scores $<24$ in heart failure patients was $51.8 \%$, which is similar to that reported by Tevik et al. (57\%) [21] for hospitalized patients with heart failure, based on the NRS-2002 screening tool.

When disease worsens, heart failure patients may progress to cardiac cachexia, characterized by sarcopenia and muscle wasting [22]. Numerous studies have recognized and highlighted malnutrition as a marker for unfavourable prognosis [22-24]. An "obesity paradox" has been described in heart failure patients, with obese or overweight patients having lower all-cause mortality rates [25]. However, in heart failure patients, oedema may mask decreases in

Table 3. Multivariate predictors of malnutrition.

\begin{tabular}{lcc} 
& Odds ratio (95\%Cl) & p \\
Heart failure & & \\
$\quad$ Mini nutritional assessment $<24$ & & \\
Female gender & $2.49(1.09-5.67)$ & 0.030 \\
CIRS severity (by 0.1) & $1.16(1.01-1.32)$ & 0.030 \\
Cardiac surgery & & \\
$\quad$ Predictors of albumin $<3.5 \mathrm{~g} / \mathrm{dl}$ & & \\
Age (by 10 years) & $1.39(1.05-1.82)$ & 0.019 \\
Days from cardiac surgery (by 10 days) & $0.46(0.31-0.69)$ & $<0.001$ \\
6MWT not perfomed & $2.25(1.32-3.85)$ & 0.003 \\
\hline Mini nutritional assessment <24 & & \\
$\quad$ Early complication after surgery & $2.37(1.15-4.90)$ & 0.020 \\
BMI (by 1 kg/m $\left.{ }^{2}\right)$ & $0.91(0.86-0.96)$ & 0.002 \\
6MWT not perfomed & $2.08(1.25-3.48)$ & 0.005 \\
\hline
\end{tabular}

lean mass, rendering the BMI an unreliable marker of undernutrition. Moreover, a lower BMI does not predict all-cause mortality in heart failure patients independently of other nutritional indicators [26].
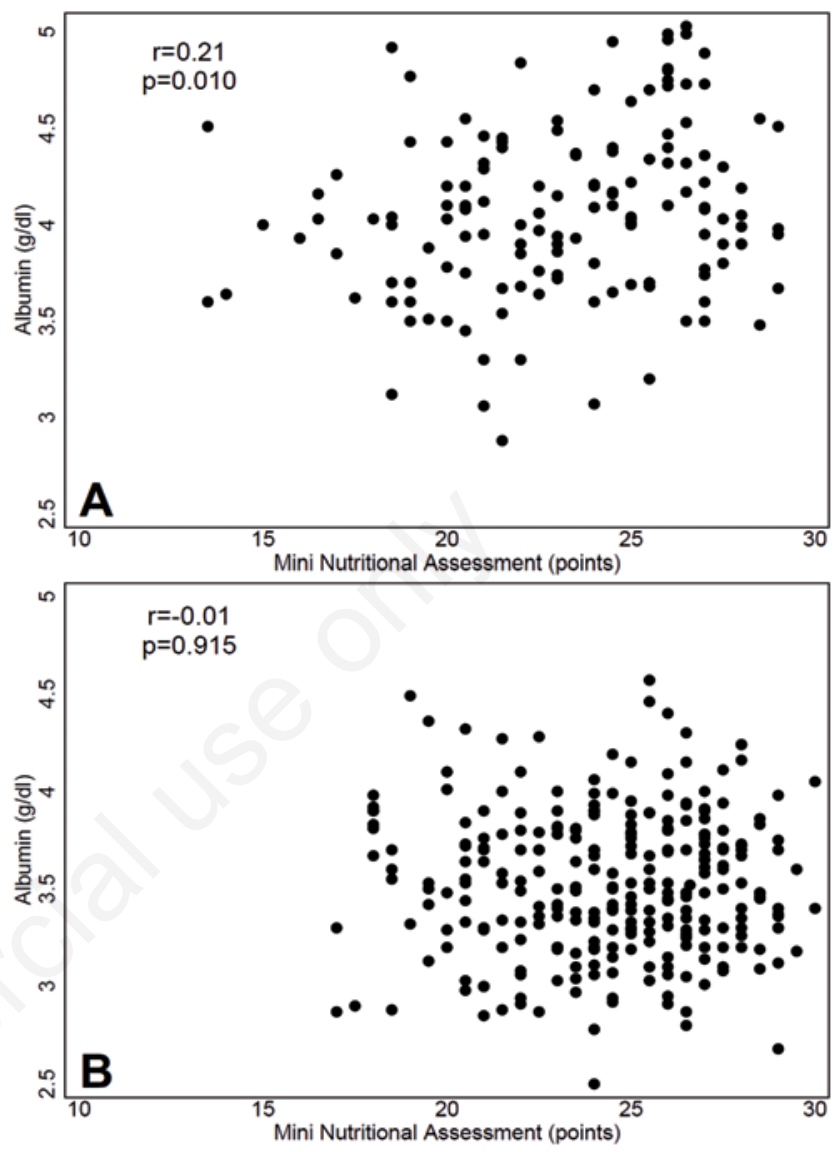

Figure 1. Scatter plots showing the correlation between MNA scores and albumin levels. A) Heart failure patients. B) Cardiac surgery patients.

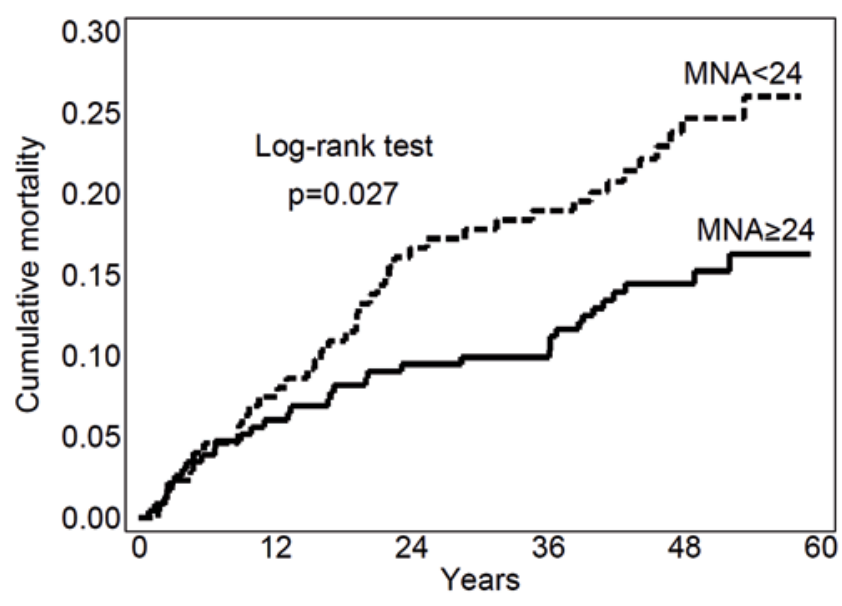

Figure 2. Kaplan-Meier curves for all-cause mortality of patients with respect to albumin levels for MNA scores $<24$ versus $\geq 24$ points. 


\section{Cardiac surgery patients}

The reported prevalence of preoperative malnutrition in cardiac surgery patients ranges from $1.2 \%$ to $46.4 \%$ [27-29]. Patients with malnutrition before cardiac surgery have increased postoperative mortality rates, hospital stay durations and complication rates. Chermesh et al. [30] evaluated the nutritional status of patients before cardiac surgery by means of the Malnutrition Universal Screening Tool (MUST). In their study, $17.9 \%$ of patients were at risk for malnutrition before surgery, and these patients had elevated mortality and morbidity rates in the postoperative phase. However, there is a lack of data regarding the prevalence of malnutrition in the postoperative phase.

\section{Albumin as a nutritional marker}

The usefulness of albumin as a nutritional marker remains controversial because many disease processes can modify its plasmatic concentration. In our study, only $6.3 \%$ of heart failure patients had albumin levels $<3.5 \mathrm{~g} / \mathrm{dl}$ versus $48.9 \%$ in the cardiac surgery group.

Racca et al. found albumin levels below the normal range in $84 \%$ of 50 nondiabetic patients admitted to in-hospital rehabilitation after cardiac surgery [31]. Albumin is a negative-phase reactive protein, and its pool is modified by inflammatory status due to infections, burning, neoplasm or surgery. However, there is no evidence supporting the use of albumin to evaluate nutritional status beyond inflammation.

In a meta-analysis of 63 studies that evaluated the effect of fasting on serum albumin levels in healthy subjects, albumin values remained normal until extreme levels of starvation were reached (i.e., BMI <12) or after more than 12 weeks of fasting [32]. In patients with heart failure, inflammation may be relevant only in the advanced and terminal phases of the disease, when malnutrition evolves into cardiac cachexia. The low percentage of cachectic patients in our heart failure cohort may explain the low incidence of hypoalbuminemia observed in this work. Patients with MNA scores $<24$ had increased mortality rates, independent of age, sex or study group. Malnutrition may be a marker of more advanced disease or frailty or may be a causative factor, causing, for example, a reduced immunity response.

\section{Clinical implications}

From the cardiologist perspective, nutritional evaluation in patients admitted to a cardiac rehabilitation programme traditionally addresses the correction of cardiovascular risk factors via lifestyle modifications. Patients surviving myocardial infarction or coronary artery revascularization receive professional counselling on diet modifications in order to reduce saturated fatty acid intake, lower plasma cholesterol levels and hinder obesity and sodium intake.

By contrast, for patients admitted to an intensive in-hospital rehabilitation programme, nutritional assessment should be performed to identify malnourished subjects that require an increased caloric supply. Changes in the epidemiology of cardiovascular disease, such as age (i.e., cardiac surgery patients are typically older) and the rapidly increasing prevalence of heart failure, have increased the number of possible recipients for intensive in-hospital rehabilitation.
For in-hospital cardiac rehabilitation, an accurate screening for malnutrition or risk of malnutrition is recommended. Albumin levels are not adequate to evaluate the nutritional status of heart failure patients. Moreover, special attention is needed for women, elderly patients and patients with low mobility or high levels of comorbidity.

As future work, further studies could evaluate whether nutritional support, guided by the proposed stratification, could improve clinical and functional outcomes.

\section{References}

1. Jensen GL, Bistrian B, Roubenoff R, Heimburger DC. Malnutrition syndromes: a conundrum vs continuum. JPEN J Parenter Enteral Nutr 2009;33:710-6.

2. ASPEN Board of Directors and the Clinical Guidelines Task Force. Guidelines for the use of parenteral and enteral nutrition in adult and pediatric patients. JPEN J Parenter Enteral Nutr 2002;26:1SA-138SA.

3. Malone A, Hamilton C. The Academy of Nutrition and Dietetics/the American Society for Parenteral and Enteral Nutrition consensus malnutrition characteristics: application in practice. Nutr Clin Pract 2013;28:639-50.

4. Niedert KC, American Dietetic Association. Position of the American Dietetic Association: Liberalization of the diet prescription improves quality of life for older adults in long-term care. J Am Diet Assoc 2005;105:1955-65.

5. Dávalos A, Ricart W, Gonzalez-Huix F, et al. Effect of malnutrition after acute stroke on clinical outcome. Stroke 1996;27:1028-32.

6. Davis JP, Wong AA, Schluter PJ, et al. Impact of premorbid undernutrition on outcome in stroke patients. Stroke 2004;35:1930-4.

7. Fuhrman MP, Charney P, Mueller CM. Hepatic proteins and nutrition assessment. J Am Diet Assoc. 2004;104:1258-64.

8. Correia MI, Waitzberg DL. The impact of malnutrition on morbidity, mortality, length of hospital stay and costs evaluated through a multivariate model analysis. Clin Nutr 2003;22:235-9.

9. Guerra RS, Sousa AS, Fonseca I, et al. Comparative analysis of undernutrition screening and diagnostic tools as predictors of hospitalisation costs. J Hum Nutr Diet 2016;29:165-7.

10. Guigoz Y, Vallas BJ, Garry PJ. Mini Nutritional Assessment: a practical assessment tool for grading the nutritional state of elderly patients. Facts Res Gerontol 1994;4:s15-59.

11. Guigoz Y, Lauque S, Vellas BJ. Identifying the elderly at risk for malnutrition. The Mini Nutritional Assessment. Clin Geriatr Med 2002;18:737-57.

12. Vellas B. Guigoz Y, Garry PJ, et al. The Mini Nutritional assessment (MAN) and its use in grading the nutritional state to elderly patients. Nutriton 1999;15:116-22.

13. Kuzuya M1, Izawa S, Enoki H, et al. Is serum albumin a good marker for malnutrition in the physically impaired elderly? Clin Nutr 2007; 26:84-90.

14. World Health Organization. Haemoglobin concentrations for the diagnosis of anaemia and assessment of severity. Vitamin and mineral nutrition information system. Available from: http://www.who.int/vmnis/indicators/haemoglobin

15. ATS Committee on Proficiency Standards for Clinical Pulmonary Function Laboratories. ATS statement: guidelines for the six-minute walk test. Am J Respir Crit Care Med 2002;166:111-7. 
16. Mahoney FI, Barthel DW. Functional evaluation: the BARTHEL Index. Md State Med J 1965;14:61-5.

17. EuroQol Group. EuroQol - a new facility for the measurement of health-related quality of life. Health Policy 1990;16:199-208.

18. Whynes DK, TOMBOLA Group. Correspondence between EQ-5D health state classifications and EQ VAS scores. Health Qual Life Outcomes 2008;6:94.

19. Miller MD, Paradis CF, Houck PR, et al. Rating chronic medical illness burden in geropsychiatric practice and research: application of the Cumulative Illness Rating Scale. Psychiatry Res 1992;41:237-48.

20. Louw JA, Werbeck A, Louw ME, et al. Blood vitamin concentrations during the acute-phase response. Crit Care Med.1992; 20:934-41.

21. Tevik K, Thürmer H, Husby MI et al. Nutritional risk screening in hospitalized patients with heart failure. Clin Nutr 2015;34:257-64.

22. Rahman A1, Jafry S, Jeejeebhoy K et al. Malnutrition and cachexia in heart failure. JPEN J Parenter Enteral Nutr 2016;40:475-86.

23. Aziz EF, Javed F, Pratap B, et al. Malnutrition as assessed by nutritional risk index is associated with worse outcome in patients admitted with acute decompensated heart failure: an ACAP-HF data analysis. Heart Int 2011;6:e2.

24. Narumi T, Arimoto T, Funayama A, et al.Prognostic importance of objective nutritional indexes in patients with chronic heart failure. J Cardiol 2013;62:307-13.

25. Curtis JP, Selter JG, Wang Y, et al. The obesity paradox: body mass index and outcomes in patients with heart failure. Arch Intern Med 2005;165:55-61. Erratum in Arch Intern Med 2008; 168:567.

26. Colín-Ramírez E, Orea-Tejeda A, Castillo-Martínez L, et al. Malnutrition syndrome, but not body mass index, is associated to worse prognosis in heart failure patients. Clin Nutr 2011;30:753-8.

27. Van Venrooij LM, Visser M, de Vos R, et al. Cardiac surgeryspecific screening tool identifies preoperative undernutrition in cardiac surgery. Ann Thorac Surg 2013;95:642-7.

28. van Venrooij LM, de Vos R, Borgmeijer-Hoelen M, et al. Preoperative unintended weight loss and low body mass index in relation to complications and length of stay after cardiac surgery. Am J Clin Nutr 2008;87:1656-61.

29. van Venrooij LM, van Leeuwen PA, Hopmans W, et al. Accuracy of quick and easy undernutrition screening toolsShort Nutritional Assessment Questionnaire, Malnutrition Universal Screening Tool, and modified Malnutrition Universal Screening Tool - in patients undergoing cardiac surgery. J Am Diet Assoc 2011;111:1924-30.

30. Chermesh I, Hajos J, Mashiach T, et al. Malnutrition in cardiac surgery: food for thought. Eur J Prev Cardiol 2014;21:475-83.

31. Racca V, Castiglioni P, Ripamonti V, et al. Nutrition markers in patients after heart surgery. JPEN J Parenter Enteral Nutr 2010; 34:143-50.

32. Lee JL, Oh ES, Lee RW, Finucane TE. Serum albumin and prealbumin in calorically restricted, nondiseased individuals: A systematic review. Am J Med 2015;128:1023.e1-22. 